\title{
Double Lid Driven Cavity with Different Moving Wall Directions for Low Reynolds Number Flow
}

\author{
Kajal Chandra Saha ${ }^{1, ~ *, ~ G o u t a m ~ S a h a ~}{ }^{2}$, Doli Rani Pal ${ }^{2}$ \\ ${ }^{1}$ Department of Applied Mathematics, University of Dhaka, Dhaka, Bangladesh \\ ${ }^{2}$ Department of Mathematics, University of Dhaka, Dhaka, Bangladesh \\ Email address: \\ kcsaha@du.ac.bd (K. C. Saha), gsahamath@du.ac.bd (G. Saha), doli.math@du.ac.bd (D. R. Pal) \\ ${ }^{*}$ Corresponding author
}

\section{To cite this article:}

Kajal Chandra Saha, Goutam Saha, Doli Rani Pal. Double Lid Driven Cavity with Different Moving Wall Directions for Low Reynolds Number Flow. International Journal of Applied Mathematics and Theoretical Physics. Vol. 4, No. 3, 2018, pp. 67-72.

doi: 10.11648/j.ijamtp.20180403.11

Received: August 14, 2018; Accepted: August 30, 2018; Published: October 10, 2018

\begin{abstract}
In this paper, a numerical examination used to analyze the flow and heat transfer characteristics inside a double lid-driven cavity underneath buoyancy consequences of thermal diffusion. The lid is due to the movement of the isothermal vertical sidewalls of different constant temperatures, while other walls are kept adiabatic. Also, the upright walls are moving at a constant rate and four different moving wall directions are considered along these walls. Further, the governing equations of the flow and thermal fields are transformed into dimensionless equations and then solved numerically using finite difference method. A contrast of the current learn is additionally carried out with the formerly published works and observed excellent agreement. Moreover, the results from numerical simulations have been presented in the form of velocities and isothermal profiles, shown graphically and discussed for different Reynolds number. Result unveils that, the influence of the development of the velocity profiles in the chamber decreases with the augmentation of Re. Besides, the intensification of Reynolds number ends in forming diminution of thermal boundary layers near the heated wall. In addition, the maximum Average Nusselt number can be obtained when the left lid poignant towards positive direction and the right lid poignant to the same direction.
\end{abstract}

Keywords: Heat Transfer, Reynolds Number, Nusselt Number, Richardson Number, Prandtl Number

\section{Introduction}

The most recent few years have seen a lot of advance in the sphere of CFD. The investigation of a lid driven flow of a fluid in a rectangular two-dimensional cavity is exceptionally compelling in see not just of the clarity of the flow geometry yet additionally the significance of the fluid flow material science. Cases of essential employments of heat exchange fluids are hydraulic heating and cooling frameworks in structures, vehicular and flying cooling frameworks in the transportation business, mechanical process warming and cooling frameworks in mash and paper, petrochemical, material, synthetic, and other preparing plants [1]. Additionally, fluid flow and heat exchange in square or rectangular cavities driven by shear and buoyancy impacts have been considered widely [2-12]. Among various geometries, the lid driven cavity flow is of extensive engrossment as it covers a spacious variety of complex hydrodynamics including distribution, distinctive vortex structure, singularity, instability, and conversion. In the reproduction of Newtonian characteristics in cavity flow postures enough difficulties and as needs be numerical strategy have been proposed by various scientists [13-14]. These examinations concentrated on fluid flows in a solitary sided lid driven cavities. In any case, fluid flow in a cavity with two sided moving lids might be found in different assembling forms, for example, covering frameworks [1516], polymer handling [17], ceramic tape casting [18], mixing [19] and drying advancements [20].

Most recently, Pal et al. [21] completed a numerical concentrated on two fold top driven pit for low Reynolds number stream utilizing limited contrast technique. Likewise, Kuhlmann et al. [22] completed test and numerical investigation of two and three-dimensional flows in rectangular cavities with anti-parallel movement of side 
walls. They found that the essential two dimensional stream is dependably not interesting as the vortex structure can change drastically with the speeds of the side walls and the aspect ratio of the cavities.

Depending on the above examinations, it is discovered that there is absence of concentrates for the entire heat exchange

Case 1:

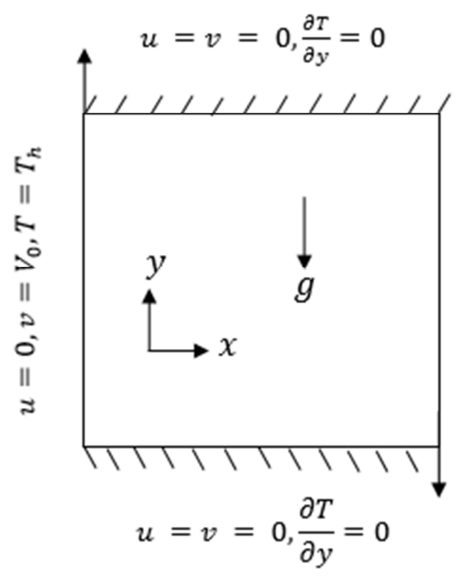

Case 3:

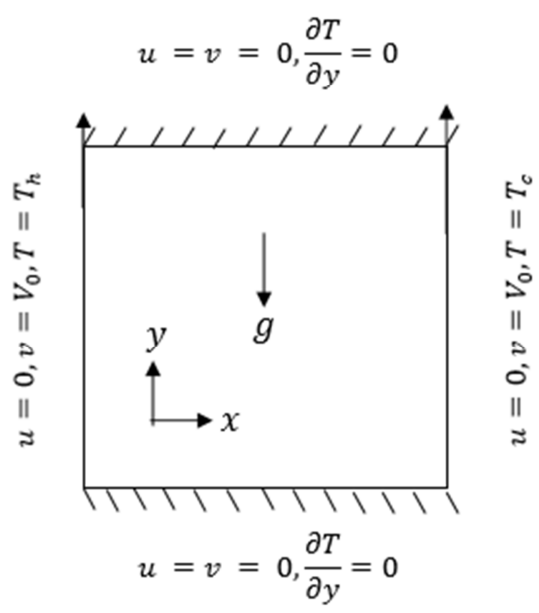

impacts in view of mixed convection for differentially heated walls with different moving walls headings [25]. Henceforth, the target of our examination is to break down the impact of the moving walls directions on heat exchange inside a two sided lid driven cavity utilizing finite difference method (FDM).

Case 2:

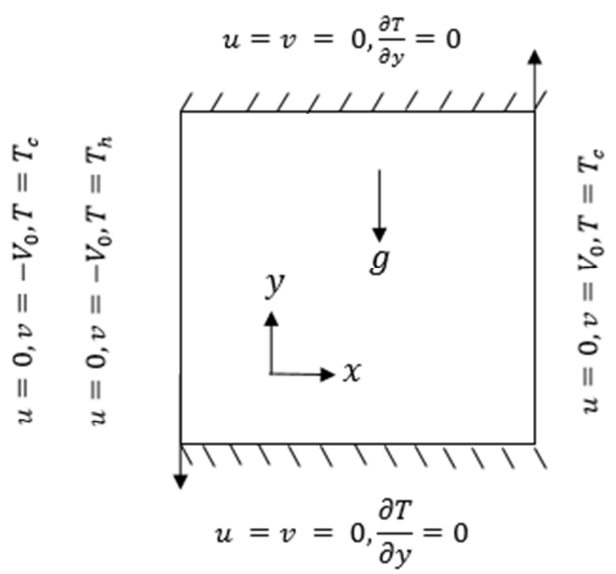

Case 4:

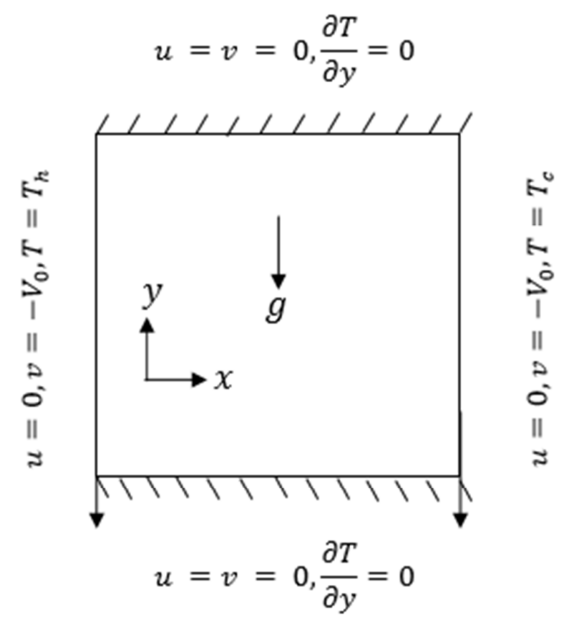

Figure 1. Schematic description of the physical model.

\section{Model Illustration and Governing Equation}

The physical model and boundary conditions are appeared in Figure 1 which is square double lid driven cavity loaded with fluid such as air. Fluid flow and heat exchange phenomena are described within the cavity where the vertical walls are moving at a steady velocity $V_{O}$ and the upper wall and bottom wall are kept up isothermal. In this study, the left and right walls are considered as hot and cold walls respectively. The fluid flow is considered to be steady and Newtonian, incompressible. The density variation is based on Boussinesq approximation. The dimensional governing equations are presented below:

$$
\begin{gathered}
\frac{\partial u}{\partial x}+\frac{\partial v}{\partial y}=0 \\
u \frac{\partial u}{\partial x}+v \frac{\partial u}{\partial y}=-\frac{1}{\rho} \frac{\partial p}{\partial x}+v\left(\frac{\partial^{2} u}{\partial x^{2}}+\frac{\partial^{2} u}{\partial y^{2}}\right) \\
u \frac{\partial v}{\partial x}+v \frac{\partial v}{\partial y}=-\frac{1}{\rho} \frac{\partial p}{\partial y}+v\left(\frac{\partial^{2} v}{\partial x^{2}}+\frac{\partial^{2} v}{\partial y^{2}}\right)+g \beta\left(T-T_{c}\right) \\
u \frac{\partial T}{\partial x}+v \frac{\partial T}{\partial y}=\alpha\left(\frac{\partial^{2} T}{\partial x^{2}}+\frac{\partial^{2} T}{\partial y^{2}}\right)
\end{gathered}
$$

Here $u$ and $v$ are the $x$ - and $y$-velocities, $p$ is the pressure and $T$ is the temperature respectively.

Also, the parameters $\rho, g, \beta$ and $\alpha$ are the fluid density, acceleration due to gravity, fluid volumetric thermal expansion coefficient and thermal diffusivity coefficient of volumetric expansion, respectively.

The dimensional boundary conditions are presented hereafter: 


$$
\left\{\begin{array}{c}
\text { Left wall: } T=T_{h} \text { at } x=0 \\
\text { Right wall: } T=T_{c} \text { at } x=L \\
\text { Bottom wall: } \mathrm{u}=0, v=0, \frac{\partial T}{\partial y}=0 \text { at } y=0 \\
\text { Top wall: } \mathrm{u}=0, v=0, \frac{\partial T}{\partial y}=0 \text { at } y=L
\end{array}\right.
$$

Also, following boundary conditions are considered for left and right walls:

Different moving wall directions:

A. Case-I: Left and right lids are moving upward and downward directions respectively

B. Case-II: Left and right lids are moving downward and upward directions respectively

C. Case-III: Left and right lids are moving upward directions

D. Case-IV: Left and right lids are moving downward directions

\section{Grid Independent Test and Validation}

In this section, details of the computer system, grid independent test and validation works have been presented and discussed.

In this paper, a in house code named DGK is developed for pre and post processing analysis and also for the simulations using Mathematica 11 with Intel ${ }^{\circledR}$ Core $^{\mathrm{TM}}$ i5-7400 CPU (3)3.00 GHz processor and 4 GB RAM. Also, grid independent test is carried out for different rectangular uniform mesh sizes and it is found that $61 \times 61$ mesh combinations shows the better performance. In addition, a broad approval work is done keeping in mind the end goal to legitimize the performance of present numerical technique and the taking care of the physical model. A decent agreement is seen with the results of Ghia et al. [2], AbuNada et al. [23], Waheed et al. [24] and Sharif et al. [8]. Elaborate description of grid independency test and validation works are presented in Pal et al. [21].

\section{Results and Discussion}

Numerical results for velocity, temperature and heat transfer of the fluid flow in a cavity with different moving wall directions are presented and discussed. An essential interest is given to the effects of heat switch for four exclusive instances as noted above. Investigations are carried out for different parameters: Richardson number $R i$ is set to 1.0 and $R e$ is varied from 100 to 1500 , Prandtl number $P r$ is set to 0.71 .

A. Case-I: Left lid is moving upward and right lid is moving downward directions

Figures 2(a, b, c) (left) present the velocity profiles for different Reynolds number. In Figure 2(a), we get the velocity profile for Reynolds number $\operatorname{Re}=100$. Here, the left (hot) wall is moving upward in direction, then the fluid which are neighborhood of the left lid becomes hot and the fluid flows in the same direction with the left wall. In velocity profile, we see that one eddy vortex formed at center of the cavity and also dominate in the whole cavity. The rotation direction of the eddy vortex is clockwise. When Reynolds number, $\operatorname{Re}$ is elevated up to $\operatorname{Re}=500$, the primary eddy vortex divided into two secondary vortices and may be the horizontal center line is separate them. With enlarge in Reynolds number $\operatorname{Re}=1500$ in Figure 2(c), we see that three eddy vortices at the core of the cavity and the middle one is dominated in the cavity. Because the Reynolds number increases, a development of two tiny inner cells at right side of the top wall and the left side of the bottom wall are observed.

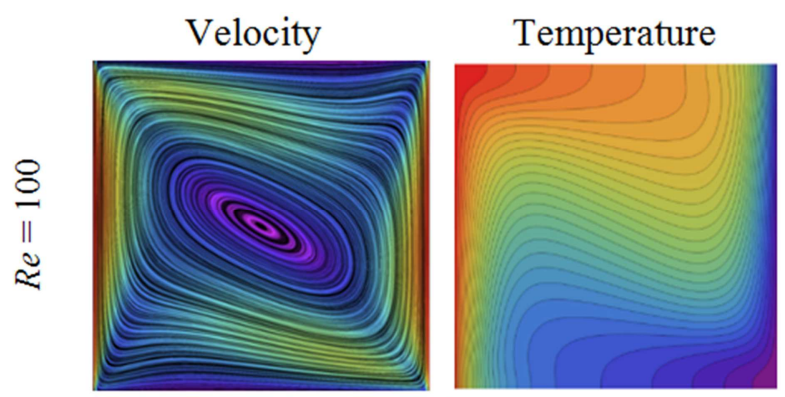

(a)

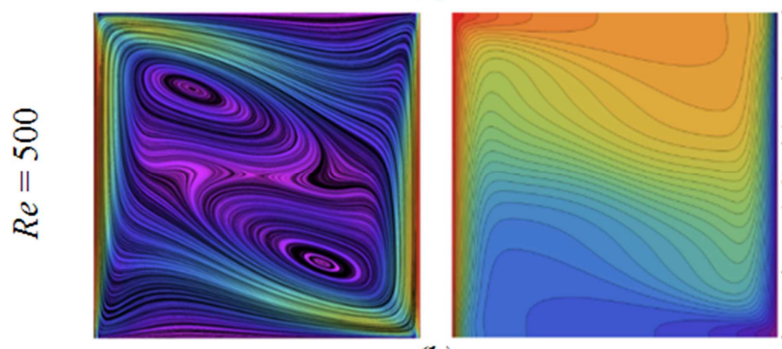

(b)

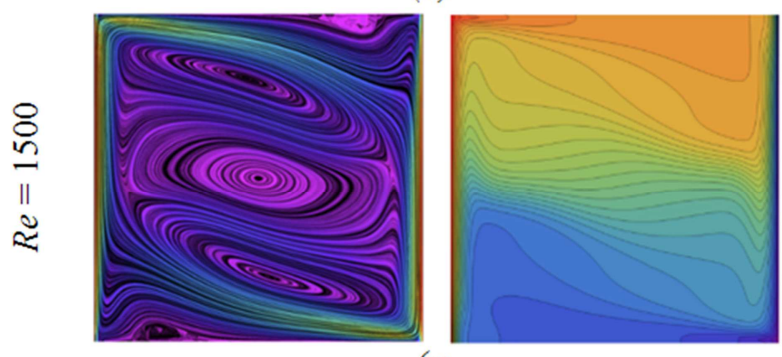

(c)

Figure 2. Deviation of velocity and temperature profiles with Re (Case I).

Figures $2(\mathrm{a}, \mathrm{b}, \mathrm{c})$ (right) present the temperature profiles for distinctive Reynolds number. It's far observed that thermal boundary layers are depicted in narrow strips adjoining to the top wall. Since the top wall was adiabatic, so the fluid is moving from left to right walls. From Figure 2(a) we see that there is some temperature difference between the left and right walls neighborhood fluids and temperature versions is almost sinusoidal. This is attributed to the increase of Reynolds number range the thermal boundary layers are reduced in Figures 2(b, c). It is apparent that at the majority of the principal areas of the cavity, fluids are properly mixed and temperature versions are very small and consequently the temperature profiles almost parallel to the horizontal wall at the core of the cavity. And the conduction performs an essential role to the augmentation of heat transfer as illustrated because of the thermal boundary layers increase at left face of the right wall. 
B. Case-II: Left and right lids are moving downward and upward directions respectively

Figures 3(a, b, c) (left) present the velocity profiles for different Reynolds number. It is observed that three vortex cells are formed initially in which two secondary vortices are seen near the side walls and the principal vortex cell is developed at the middle of the cavity. Also, all the three vortices are moving in an anticlockwise in direction. Moreover, the left side vortex is poignant from left to right side direction while the right side vortex is moving from right to left side direction. As Reynolds number increases, the secondary vortexes started to become small and the primary vortex divide into another two sub vortexes as shown in Figure3(b). When $R e$ is elevated up to $R e=1500$, robust recirculation structures are formed and the cores of the top and lower recirculation structures pass toward the left aspect of the top corners and proper aspect of the bottom corners respectively as proven in Figure 3(b). In addition, a small vortex is formed in the top-right nook of the cavity.

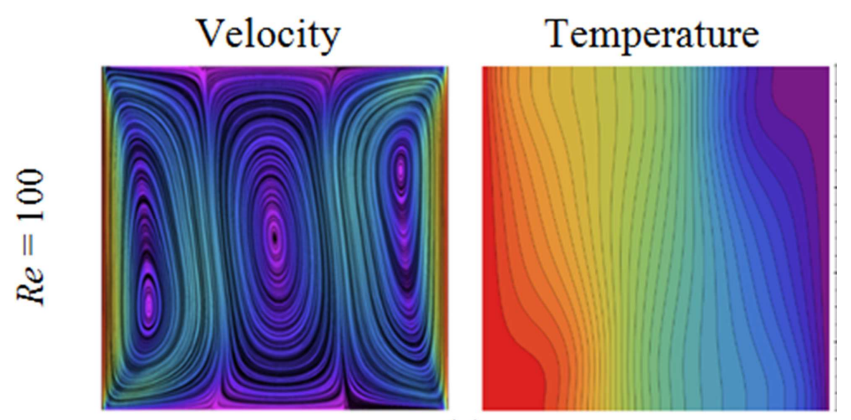

(a)

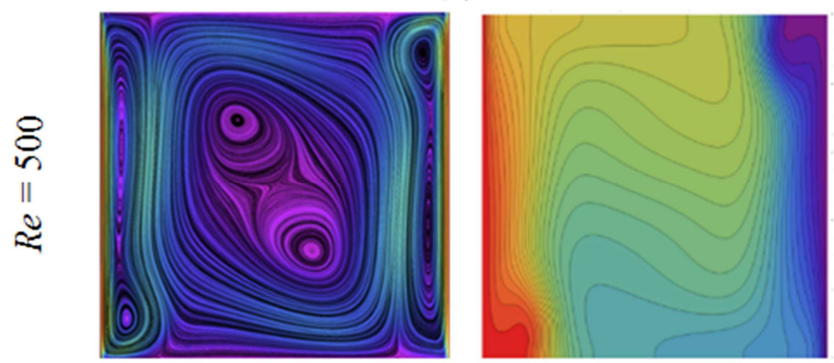

(b)

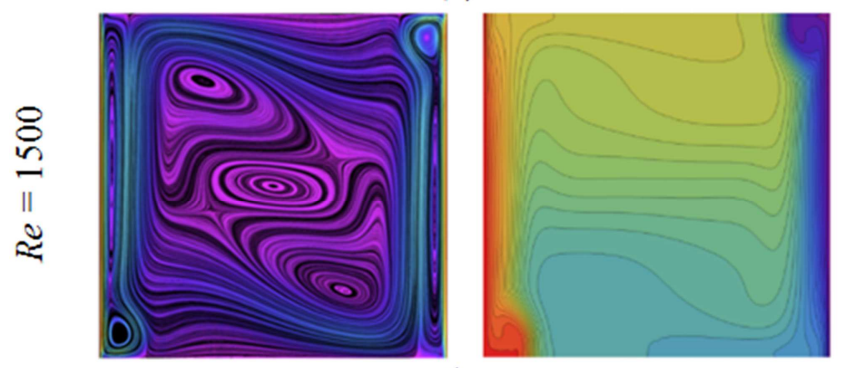

(c)

Figure 3. Deviation of velocity and temperature profiles with Re (Case II).

Figures $3(\mathrm{a}, \mathrm{b}, \mathrm{c})$ (right) present the temperature profiles for exceptional Reynolds variety. Temperature profiles demonstrated that temperature gradient close to the cold wall and especially the hot wall could be very low which indicates low rate of warmth transfer in these regions. As Reynolds number increased, the temperature is shipped all through the entire cavity. It could be understood from the results that the temperature gradient at the top wall is high this means that there may be an excessive heat transfer charge in this region. But, the temperature gradient is stronger in comparison to the preceding profile at left corner of the bottom wall.

C. Case-III: Left and right lids are moving upward directions

Figures $4(\mathrm{a}, \mathrm{b}, \mathrm{c})$ (left) present the velocity profiles for different Reynolds number. It is observed that two primary vortices are shaped near the lids due to the motion of lids in the identical directions. In keeping with the path of the lids motion, the vortex that's shaped through the force due to the motion of the left lid is clockwise and the vortex that's formed via the pressure as a result of the motion of the right lid is counter clockwise for $R e=100$. When $R e=500$, streamline behaviors in the two-dimensional double lid driven cavity discussed here could be categorized by a primary recalculating vortex covering utmost of the inside zone of the cavity produced by the moving lids and one secondary vortex close to the right wall.

Figures $4(a, b, c)$ (right) present the temperature profiles for different Reynolds number. It is seen that thermal boundary layer growth is at the top of the heated wall and also the temperature gradients are strong. So the direction of the fluid is from left to right and spread in the whole cavity. For increases of Reynolds number, the thermal boundary layer is reduced and the hot fluid dominates within the cavity.

Overall, increasing of Reynolds number, intensity of the velocity and temperature profiles near the hot wall increases and stronger circulation appears in the cavity. So the flow rate near the top surface of the left wall increases and consequently the total rate of the heat transfer is increasing.

D. Case-IV: Left and right lids are moving downward directions

Figures 5(a, b, c) present the velocity and temperature profiles for different Reynolds number. In this case, the left lid moves downward and the direction of movement of the right lid is also downward.

It is observed that there is a primary circulating cell in the center of the cavity and two tiny secondary eddies at the side walls generated by the movement of the vertical walls for $R e=100$. Buoyancy force does not play an essential role when $\mathrm{Ri}$ is much less than unity. Since the shear pressure dominates the buoyancy force, the core vicinity of the circulating eddy inner the cavity is close to the top of left wall and the backside of the right wall of the cavity. The temperature impact is visible close to the hot wall as proven in Figure 5(a) for Reynolds numbers $R e=100$. It is also found that the isotherms show the thickness of the thermal boundary layer is small and the isotherms are allotted over the total cavity for forced and mixed convection regimes. Flow pattern remains opposite for high Reynolds number of active portion with same $R i=1.0$ which is observed in Figures $4(b, c)$ but a significant change occurs for isotherms shows the cool fluid spread out in whole cavity and dominate. 


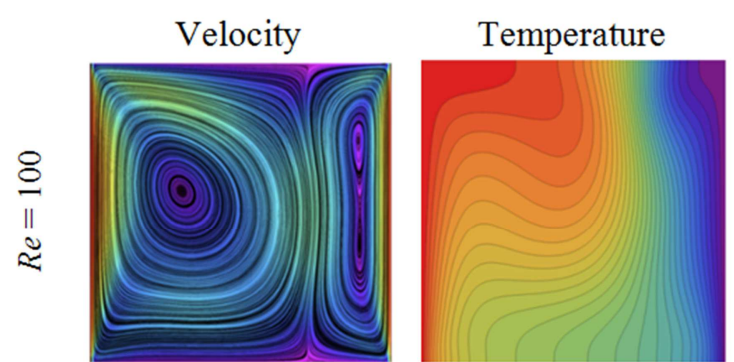

(a)

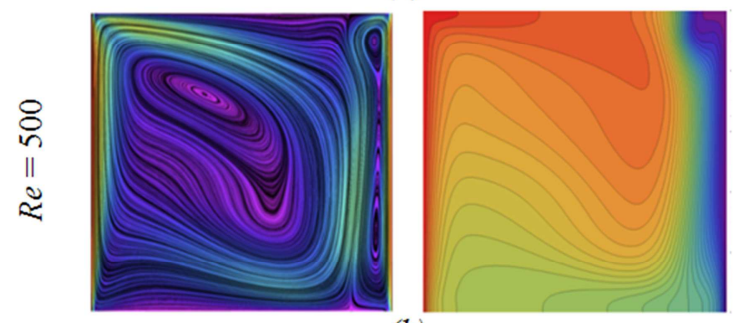

(b)

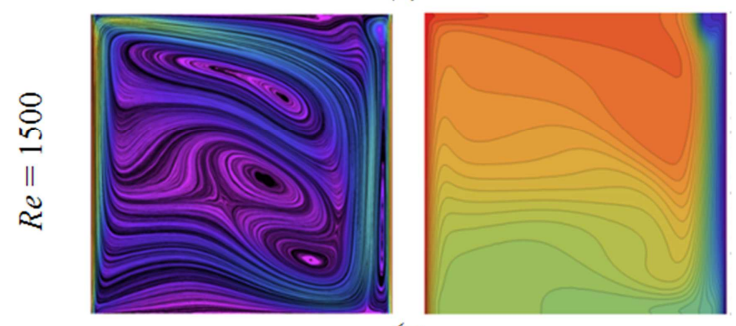

(c)

Figure 4. Deviation of velocity and temperature profiles with Re (Case III).
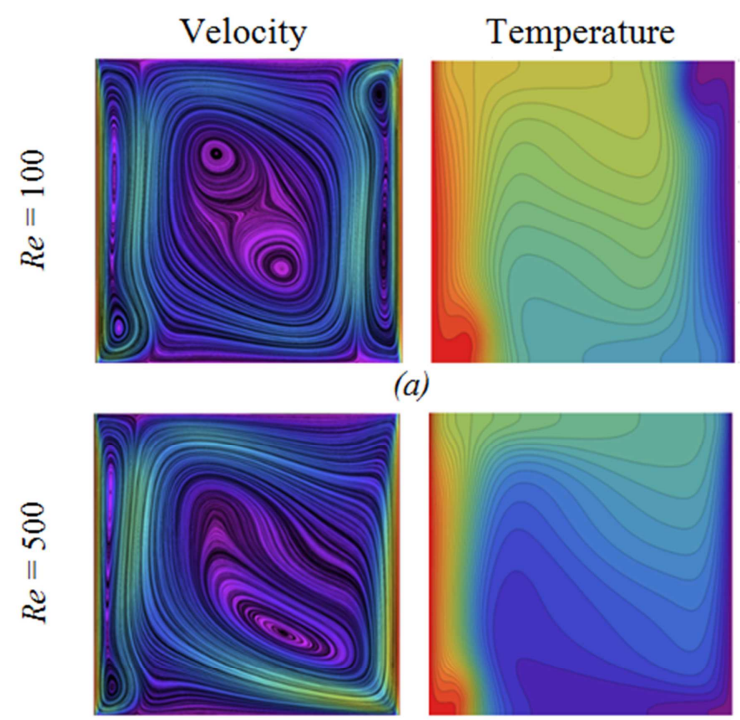

(b)

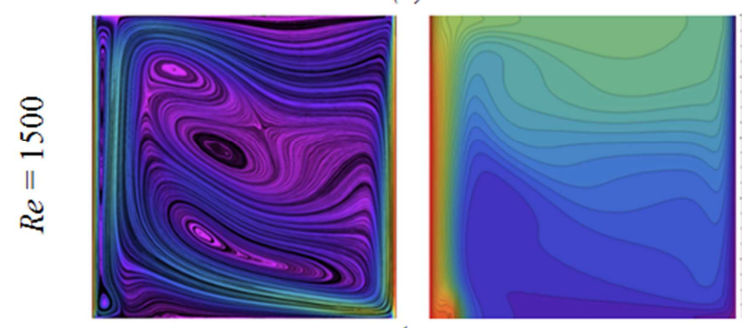

(c)

Figure 5. Deviation of velocity and temperature profiles with Re (Case IV).
Variation of the Average rate of the heat transfer with different Reynolds number are shown in Table 1 for four different cases. It's far observed that, $\overline{N u}$ increases with the increase of Reynolds number for $\operatorname{Pr}=0.71$ and $R i=$ 1.0. On increasing Reynolds number from $R e=100$ to $R e=1500$, the overall heat transfer rate rapidly increases for small Reynolds number and gradually increases for higher $R e$ in all cases. Meanwhile, the average Nusselt number is found higher for case-I while the opposite behavior is seen for case-II. In addition, the rate of average heat transfer is almost identical as observed for cases-III and IV. Such behavior is realistic as discussed in case-IV.

Table 1. Average Nu results with $\mathrm{Pr}=0.71$.

\begin{tabular}{lllll}
\hline & \multicolumn{5}{c}{ Average Nusselt Number, Nu } \\
\hline Re & Case - I & II & III & IV \\
\hline 100 & 4.59 & 1.25 & 2.11 & 2.12 \\
500 & 10.49 & 2.94 & 4.85 & 4.86 \\
1000 & 14.31 & 4.15 & 6.83 & 6.89 \\
1500 & 16.81 & 5.08 & 7.97 & 8.06 \\
\hline
\end{tabular}

\section{Conclusion}

The effect of Reynolds number flow inside a double-lid driven square cavity on combined convection within the presence of adiabatic horizontal walls was investigated in this paper. A 4th order finite difference technique is used to resolve the Eqs. (1-5) as mentioned in segment 2. The examine has been performed for Reynolds number $100 \leq$ $R e \leq 1500$. It should be note that Richardson number (Ri) and Prandtl number (Pr) are kept constant. Form the current study, it can be concluded that:

1. The strength of the velocities over the entire cavity decreases with the increment of $R e$.

2. The growth of Reynolds number ends in forming thermal boundary layers on the heated wall of the cavity.

3. The average $\mathrm{Nu}$ increases significantly with the increase of $R e$.

4. It is observed that left and right walls are moving upward directions yields the excellent warmth transfer overall performance of the considered cases.

\section{Acknowledgements}

This work has been fully supported by University Grant Commission (UGC), Bangladesh with Grant No.: Reg./Admin-3/76338 (Year: 2017-18).

\section{References}

[1] Choi, S. U. S. (1995). Enhancing thermal conductivity of fluids with nanoparticles.ASME Fluids Eng. Div., 231, 99105 .

[2] Ghia, U., Ghia, K. N., \& Shin, C. T. (1982). High-Re Solutions for Incompressible Flow Using the Navier-Stokes Equations and a Multigrid Method. J. Comput. Physics, 48, 387- 411. 
[3] Moallemi, M. K., \& Jang, K. S. (1992). Prandtl number effects on laminar mixed convection heat transfer in a liddriven cavity. Int. J. Heat Mass Transfer, 35(8), 1881- 1892.

[4] Hakan, F., \& Oztop, I. D. (2004). Mixed convection in twosided lid driven differentially heated square cavity. Int. J. Heat Mass Transfer, 47, 1761-1769.

[5] Chamkha, A. J. (2002). Hydromagnetic combined convection flow in a vertical lid-driven cavity with internal heat generation or absorption. Numerical Heat Transfer, Part A, 41, 529-546.

[6] Hussain, S. (2017). Effects of inclination angle on mixed convective nanofluid flow in a double lid-driven cavity with discrete heat sources. Int. J. Heat Mass Transfer, 106, $847-$ 860 .

[7] Sivasankaran, V. S. (2013). Numerical study on mixed convection in an inclined lid-driven cavity with discrete heating. Int. Comm. Heat Mass Transfer, 46, 112- 125.

[8] Sharif, M. A. R. (2007). Laminar mixed convection in shallow inclined driven cavities with hot moving lid on top and cooled from bottom, Appl. Therm. Eng, 27, 1036-1042.

[9] Cheng, T. S. (2011). Characteristics of mixed convection heat transfer in a lid-driven square cavity with various Richardson and Prandtl numbers. Int. J. Thermal Sciences, 50, 197- 205.

[10] Sousa, S. K. (2014). A detailed study of lid-driven cavity flow at moderate Reynolds numbers using Incompressible SPH. Int. J. Num. Methods Fluids, 76, 653- 668.

[11] Omari, R. (2016). Numerical Investigation of a Mixed Convection Flow in a Lid-Driven Cavity. American $J$. Computational Mathematics, 6, 251- 258.

[12] Adair, M. J. (2015). Developing an Understanding of the Steps Involved in Solving Navier-Stokes Equations. The Mathematica Journal, 17, 1-19.

[13] Benjamin, A. S., \& Denny, V. E. (1979). On the convergence of numerical solutions for 2-D flows in a cavity at large Re, $J$. Comput. Phys., 33, 340-358.

[14] Ghia, U., Ghia, K. N., \& Shin, C. T. (1982). High-Re solutions for incompressible flow using the Navier-Stokes equations and a multigrid method. J. Comput. Phys., 48, 387-411.
[15] Cao, Z., \& Esmail, M. N. (1995). Numerical study on hydrodynamics of short-dwell paper coaters, AIChE J., 41, $1833-1842$.

[16] Triantafillopoulos, N. G., \& Aidun, C. K. (1990). Relationship between flow instability in short-dwell ponds and cross directional coat weight non-uniformities, TAPPI J., 73, 127136.

[17] Gaskell, P. H., Summers, J. L., Thompson, H. M., \& Savage, M. D. (1996). Creeping flow analyses of free surface cavity flows, Theoret. Comput. Fluid Dynam., 8, 415-433.

[18] Hellebrand, H. Tape Casting, in: R. J. Brook (Ed.), Processing of Ceramics, Part1, VCH Verlagsgesellschaft mbH, 17A, Weinheim, 1996, 190-265.

[19] Leong, C. W., \& Ottino, J. M. (1989). Experiments on mixing due to chaotic advection in a cavity. J. Fluid Mech., 209, 463499.

[20] Alleborn, N., Raszillier, H., \&Durst, F. (1999). Lid-driven cavity with heat and mass transport. Int. J. Heat Mass Trans., $42,833-853$.

[21] Pal, D. R., Saha, G., \& Saha, K. C. (2018). A case study of double lid driven cavity for low Reynolds number flow. Dhaka University J. Science, 66(2), 95-101.

[22] Kuhlmann, H. C., Wanschura, M., \& Rath, H. J. (1997). Flow in two-sided lid-driven cavities: non-uniqueness, instabilities, and cellular structures. J. Fluid Mech., 336, 267-299.

[23] Abu-Nada, E., \& Chamkha, A. J. (2010). Mixed convection flow in a lid-driven inclined square enclosure filled with a nanofluid. European Journal of Mechanics B Fluids, 29, 472482 .

[24] Waheed, M. A. (2009). Mixed convective heat transfer in rectangular enclosures driven by a continuously moving horizontal plate. Int. J. Heat Mass Transfer, 52, 5055-5063.

[25] Malik, S., \& Nayak, A. K. (2016). A comparative study of mixed convection and its effect on partially active thermal zones in a two sided lid-driven cavity filled with nanofluid. Engineering Science and Technology, an International Journal, 19, 1283-1298. 